\title{
Large wetted areas of divertor power loads at Wendelstein 7-X
}

\author{
H. Niemann, ${ }^{1,2}$, a) P. Drewelow, ${ }^{1}$ M. W. Jakubowski, ${ }^{1}$ A. Puig Sitjes, ${ }^{1}$ B. Cannas, ${ }^{3}$ Y. Gao, ${ }^{1}$ F. Pisano, ${ }^{3}$ R. \\ König, ${ }^{1}$ R. Burhenn, ${ }^{1}$ P. Hacker, ${ }^{1}$ F. Reimold, ${ }^{1}$ D. Zhang, ${ }^{1}$ K. J. Brunner, ${ }^{1}$ J. Knauer, ${ }^{1}$ T. Sunn Pedersen, ${ }^{1,2}$ and \\ W7-X Team ${ }^{1}$ \\ 1) Max-Planck-Institut für Plasmaphysik, Wendelsteinstr. 1, 17491 Greifswald, \\ Germany \\ ${ }^{2)}$ Universität Greifswald, Greifswald, Germany \\ 3) University of Cagliari, Cagliari, Italy
}

(Dated: 13 July 2020)

Wendelstein 7-X(W7-X), an optimized stellarator, conducted its first divertor operation. It is using an island divertor concept for the heat and particle exhaust. Large wetted areas of up to $1.5 \mathrm{~m}^{2}$ have been reached, showing the benefits of the divertor concept and very efficient heat flux spreading. Moreover, a positive scaling of the wetted area with increasing SOL power is observed, which is important for operations at high input power. A definition of the wetted area is made based on thermographic camera observations for the 3D structure of W7-X with comparability to the definitions of other machines (e.g., tokamaks).

Keywords: Wendelstein 7-X, divertor, wetted area, THEODOR, heat flux, stellarator

Any future reactor based on the concept of magnetically confined plasma needs to face a challenge of handling potentially excessive heat loads, leaving the confined plasma volume through a narrow region called the scrape-off layer (SOL). It is a part of the magnetic topology at the outermost boundary, where open field lines intersect a material surface. Heat and particles are transported along the field lines towards a so-called divertor, where they are removed in a controlled way. It is expected that in ITER, about $100 \mathrm{MW}$ of the power will enter into the $\operatorname{SOL}\left(P_{S O L}\right)$ from the main plasma ${ }^{2}$. Due to the narrow width ${ }^{3}$ of the SOL $\left(\lambda_{q} \sim 0.9 \mathrm{~mm}\right)$, ITER expects very high values of the parallel heat flux (of up to $1 \mathrm{GW} / \mathrm{m}^{2}$ ). The narrow SOL in tokamaks results in part from its geometry, namely short connection length $L_{\mathrm{c}} \sim \mathcal{O}(10)[\mathrm{m}]$ along the magnetic field lines between intersecting material surfaces. Short $L_{c}$ with a very small ratio of perpendicular to a parallel heat transport $\left(\chi_{\perp} / \chi_{\|} \sim 10^{-6}\right)$ leads to a narrow SOL. In a stellarator with an island divertor ${ }^{4}$, both factors are somewhat improved: magnetic field lines in SOL have a connection length $L_{\mathrm{c}} \sim \mathcal{O}(100)[\mathrm{m}]$, and the perpendicular component of the heat transport coefficients contributes stronger in the modeling ${ }^{5}$ of the plasma boundary. As it will be shown in this work, both lead to a significant increase of the wetted area on a divertor surface in the stellarator Wendelstein 7-X (W7-X). Moreover we observe a positive scaling of wetted area with increasing $P_{\mathrm{SOL}}$, i.e. an increase of $P_{\mathrm{SOL}}$ from $1 \mathrm{MW}$ to $4 \mathrm{MW}$ yields increase of wetted area by more than a factor of two from $0.6 \mathrm{~m}^{2}$ to $1.5 \mathrm{~m}^{2}$.

W7-X is an optimized modular stellarator ${ }^{6}$ operating with an island divertor. The first divertor campaign started in 2017. The device has a five-fold symmetry and large magnetic islands at the plasma edge intersecting with the divertor (hence its name: island divertor).

\footnotetext{
a)Holger.Niemann@ipp.mpg.de
}

In its initial divertor campaign, $\mathrm{W} 7-\mathrm{X}$ was equipped with ten, toroidally localized, inertially cooled graphite divertor units. In the so-called standard configuration, five independent magnetic islands located at the $t=1$ surface form five independent helical regions interacting with one upper and one lower divertor. In Fig. 1(b) a poloidal cross-section of three out of five magnetic islands is presented.

Each of the divertor units is observed by a combined visible and infrared (IR) system ${ }^{7}$. Nine of them are equipped with a microbolometer infrared camera working at a spectral range of $8-10 \mu \mathrm{m}$, and observing the divertor surface directly via a wide-angle lens. A tenth observation system was a prototype endoscope with a semi-conductor infrared camera working at a spectral range of 3-5 $\mu \mathrm{m}$, and a wide-angle mirror-based optics. The incoming heat flux can be evaluated from the time evolution of the divertor surface temperature by, e.g., solving the $2 \mathrm{D}$ heat diffusion equation along a profile line $^{8}$. At W7-X, this is performed with the help of the THEODOR code $^{9}$. In order to perform calculations, temperature data had to be mapped on a computer-aided design (CAD) machine model. An example view of one of the divertors (lower in module 2) with overlaid heat flux distribution is shown in figure 1(a). Typical power deposition patterns at Wendelstein 7-X consist of elongated structures called strike lines on horizontal and vertical target plates. These are the areas of strongest plasmawall interaction, where heat and particles are deposited. In standard configuration, two strike lines are formed by intersections of the magnetic islands with target plates (see Fig. 1(b)). Figure 2(b) shows the heat flux profiles, indicated with red lines in figure 1(a). The full width half maximum (FWHM) distance for the horizontal target profile is $8.2 \mathrm{~cm}$, and the FHWM for the profile on the vertical target reaches values of about $12.2 \mathrm{~cm}$. It is obvious from figure 1(a) that the position of the profile matters for the analysis.

Thus the complete pattern has to be analyzed. There- 


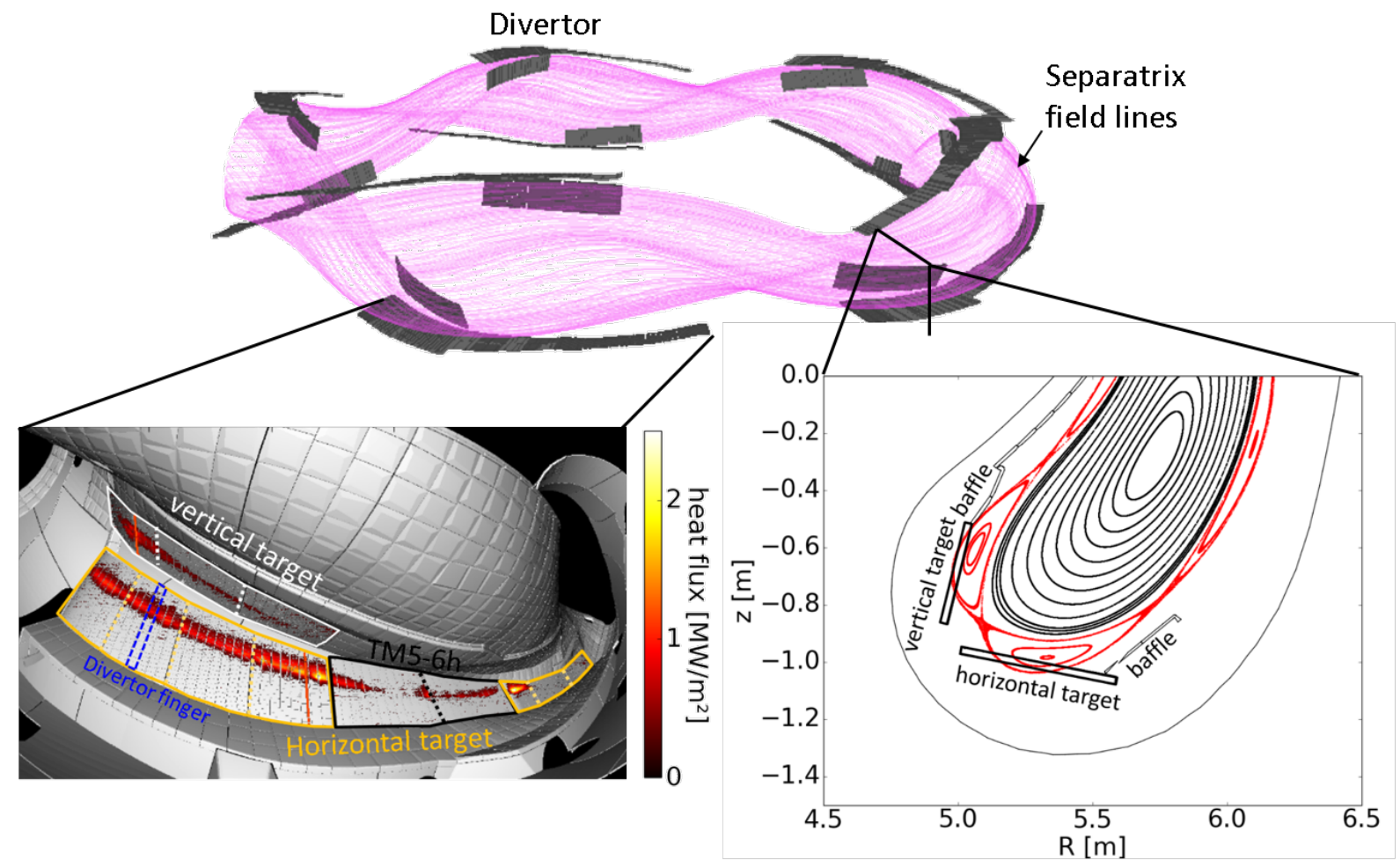

(a) IR camera view into the torus

(b) Poincare plot, Standard Configuration

FIG. 1. The shape of W7-X plasma (pink) and ten divertor units in gray. (a) Heat flux distribution on a divertor surface as measured by an infrared camera during a discharge \#20171109.021, $t=1 \mathrm{~s}$. Heat flux values are back-projected into the camera image and are overlaid onto a CAD model of the divertor. Two red lines (on the vertical and horizontal target) indicate location used to visualize heat flux profiles in Fig. 2(b). (b) Poincare plot of the lower half of the vessel at the toroidal angle of $\phi=132^{\circ}$. The plane is located in the middle of the low iota target. Five resonant magnetic islands form five X-Points and the separatrix (colored in red).

fore the divertor is structured into target modules (TM) (see dotted border lines in figure 1a) and each TM into stacks of graphite blocks, called fingers, which are poloidally aligned (see blue marked region in figure 1a). Up to 15 profiles are defined on each finger to evaluate the incoming heat flux from the evolution of the surface temperature with the THEODOR code ${ }^{10}$. A toroidal distance among the profiles is ca. $3 \mathrm{~mm}$.

To characterize the load pattern in the standard configuration of Wendelstein 7-X, the maximum heat flux and the wetted area are discussed. The first one is merely the maximum of all the profiles, while the latter one has to be defined. In tokamak research, the wetted area is defined as ${ }^{11,12}$ :

$A_{\text {wet }}=\frac{P_{d i v}}{q_{d i v}^{m a x}}=2 \pi R_{d i v} f_{\text {tor }} \frac{\int q_{d i v}(r) d r}{q_{d i v}^{m a x}}=2 \pi R_{\text {div }} f_{\text {tor }} \lambda_{\text {int }} f_{x}$

with $\mathrm{P}_{d i v}$ the divertor load, $R_{d i v}$ being the major radius of the device, $f_{\text {tor }}$ the percentage of toroidal circumference receiving heat load, $f_{x}$ the flux expansion factor, $\lambda_{\text {int }}$ the integral power fall-off length of the target heat flux profile, $q_{d i v}(r)$ the radial heat flux profile and $q_{d i v}^{\max }$ the maximum of the heat flux profile. Formula 1 can be structured in a toroidal part $\left(2 \pi R_{\text {div }} f_{\text {tor }}\right)$ and a poloidal component, the strike-line width $\left(\int q_{d i v}(r) d r / q_{d i v}^{\max }=\right.$ $\left.\lambda_{\text {int }} f_{x}\right)$

This definition includes the assumption of toroidal symmetry with a toroidally circumferential divertor for a tokamak. For a stellarator with discrete divertor units, the wetted area must be derived from the whole heat load pattern from all divertors. Additionally, drift effects ${ }^{13}$ result in different heat and particle fluxes on upper and lower divertors. The poloidal component, the strike-line width, can be calculated for W7-X in the same way, but need to be extended to one profile per divertor finger. In this way, changes of the width in toroidal direction are taken into account. The central profile of each finger is used to avoid the effect of leading edges. Each profile is multiplied with the width of the finger to get the wetted area on the finger. The toroidal component is therefore split up in a sum over all divertors and a sum over all wetted areas of a divertor. A mean upper and a mean lower divertor heat flux distribution $\langle q\rangle_{j}$ is calculated to simplify this analysis and to reduce the effects of hot spots and artifacts. Including the periodicity of W7-X 


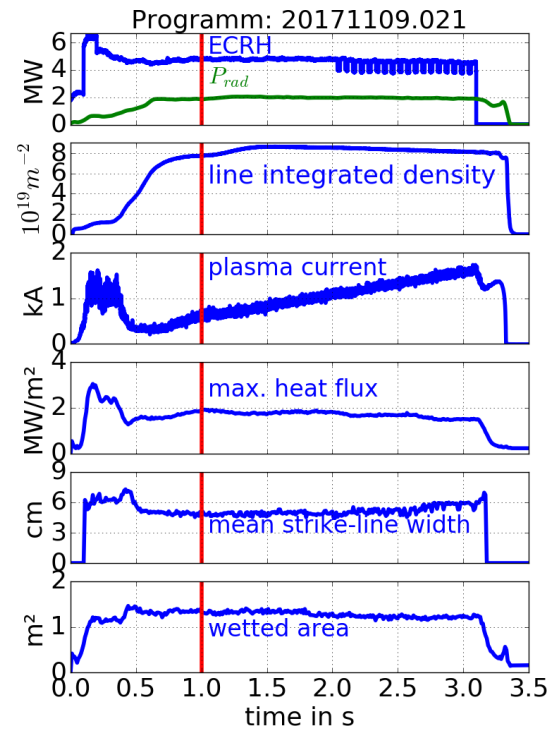

(a) Overview

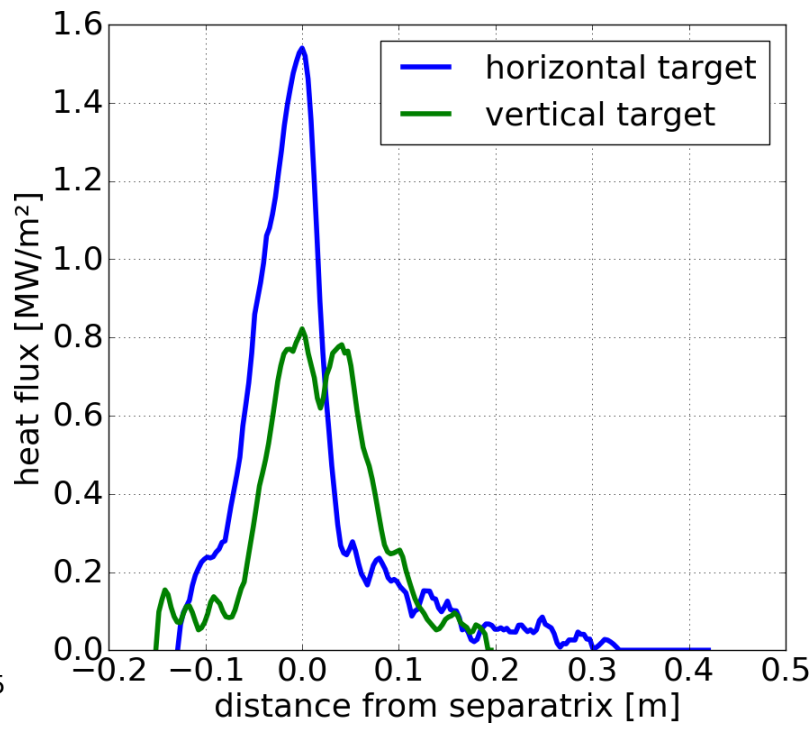

(b)Heat flux profiles

FIG. 2. (a) Overview of the main plasma parameters and maximum heat flux, mean strike-line width, and wetted area for one experiment program in the standard configuration (b) Heat flux profiles from the vertical and horizontal target for a discharge in standard configuration of the lower divertor in module 2. The positions of the profiles are indicated in figure 1 (a) by the red lines. The profile on the horizontal target FWHM of $8.2 \mathrm{~cm}$, while the profile on the vertical target reaches a FWHM value of $12.2 \mathrm{~cm}$. Program 20171109.021, 1s

$(=5)$ gives the following equation:

$$
A_{\text {wet }}=5 \sum_{j}^{\text {upper/lower }} \sum_{i=1}^{\# \text { finger }} \frac{\int_{s}<q(s)>_{i j} d s}{\max \left(<q>_{j}\right)} w_{i}
$$

with $\mathrm{j}$ standing for data from the upper or lower divertor, $\mathrm{i}$ is the index of the divertor finger, $\mathrm{s}$ is the coordinate along profiles and the length of a finger, $\mathrm{w}_{i}$ for the width of a finger $\mathrm{i}$ and $q_{i j}(s)$ for the central heat flux on finger $i$ of the heat flux distribution of the average divertor $j$ and $\max \left(<q>_{j}\right)$ for the maximum heat flux in the heat flux distribution of the average divertor $\mathrm{j}$ (mean upper or lower divertor). In this way, hot spots and leading edges are not affecting the results, and the up-down asymmetry is taken into account.

A set of 60 different programs in the standard configuration with helium gas fueling are analyzed to investigate the changes of maximum heat flux and wetted area for different plasma conditions. Figure 2(a) shows an overview plot for a typical plasma program in W7-X. From each program, data is taken from specific timepoints, to avoid plasma start-up and plasma collapse effects. Such a time-point is represented with the red line in the overview plot.

All these programs have been conducted with on-axis electron cyclotron resonance heating $(\mathrm{ECRH})$ with injected powers of 1 to $5 \mathrm{MW}$. Also, the line integrated density has been varied between 1 and $9^{*} 10^{19} \mathrm{~m}^{-2}$. As a consequence of the combination of different heating powers and densities, the set also covered a broad range of radiated powers. The fraction of radiated power spans from less than $10 \%$ up to nearly $50 \%$ (see figure 3 bottom).

Figure 3 shows the changes of the wetted area for the 60 programs. The top row shows the dependency on the power entering the $\mathrm{SOL}\left(\mathrm{P}_{S O L}=\mathrm{P}_{\text {input }}-\mathrm{P}_{\text {rad }, \text { Core }}\right)$, the second row the dependency on the electron density, and the last row the dependency on the radiated fraction. It has to be mentioned that the line integrated electron density is unfortunately not available for all evaluated programs, resulting in fewer points in the second row and uncolored points in the other diagrams. The expectations for the wetted area depend on the assumptions of radial transport and plasma behavior. Assuming that the wetted area depends mainly on the ratio between perpendicular and parallel heat flux and using the relation from the extended two-point model ${ }^{14}$ (equation 4) gives the following relation:

$$
A_{w} \sim \frac{q_{\perp}}{q_{\|}}=\frac{\chi_{e, i} n}{\kappa_{e, i} T_{e, i}^{5 / 2} \Theta^{2}}
$$

Two main dependencies can be seen for the wetted area for constant thermal conductivity $\kappa$ and diffusivity $\chi$. With increasing power entering the SOL, the electron temperature at the separatrix should increase, which reduces the ratio and, therefore, the wetted area. An increase of the density, on the other hand, increases the ratio and, thus, also the wetted area.

A heuristic drift model ${ }^{15}$, on the other hand, suggests that the width and, therefore, the wetted area, are affected by magnetic field drifts and scales in the following 
Standard configuration
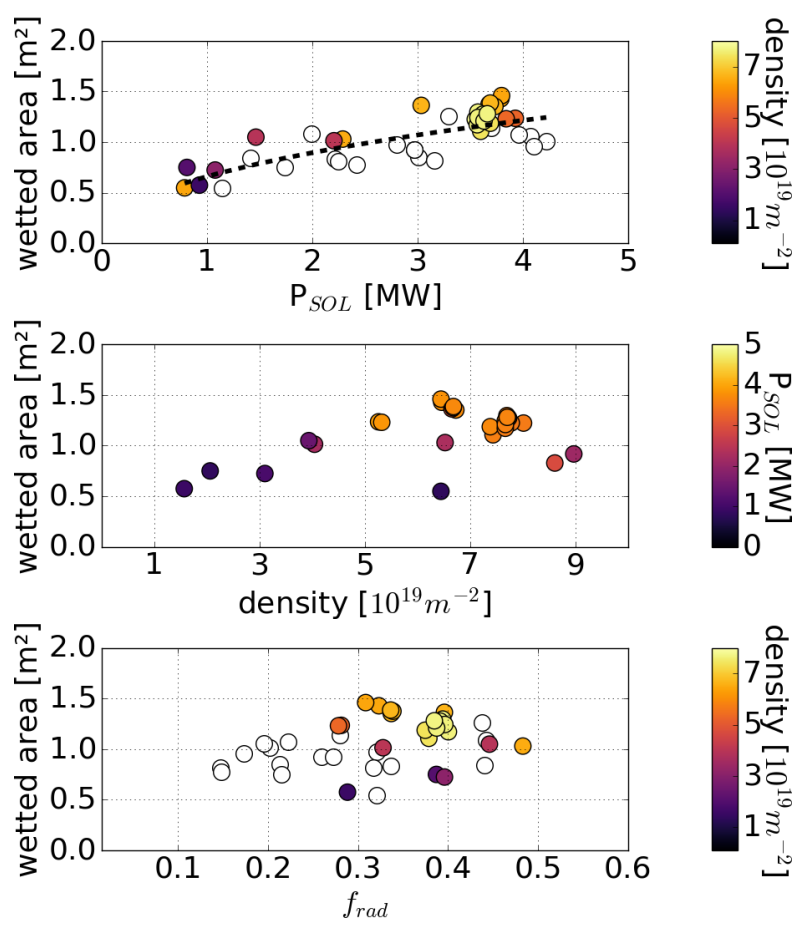

FIG. 3. Measured maximum heat flux and wetted area for 60 performed plasma programs in He in standard configuration. In the top row, the data for different heating power is shown. The second row shows the data for different line integrated densities and the last row, the data for different fractions of radiated energy.

way (from equation 5 from $^{15}$ ):

$$
A_{w} \sim \lambda_{q} \sim P_{S O L}^{1 / 8}
$$

The model predicts an increase of the width with increasing SOL power $P_{S O L}$ and no scaling with density.

The measured data for helium plasmas shows that the values of total wetted areas are reaching values from $0.5 \mathrm{~m}^{2}$ to $1.5 \mathrm{~m}^{2}$, and are increasing for increasing $\mathrm{SOL}$ power over the full density range. A power-law fit results in scaling of $\mathrm{P}_{S O L}^{0.44 \pm 0.03}\left(R^{2}=0.64\right.$ ) (see also dashed line in top graph of figure 3 ) for the dependency of the wetted area on the SOL power on the full data set.For increasing density, the general trend is not clear. Looking at density changes for similar heating powers, e.g., the orange points in the second graph of figure 3, implies no significant change of the wetted area with changing density. The same is also observed when plotting the wetted area as a function of radiated fraction, no clear relation between both quantities.

Similar values for the wetted area of up to $1.68 \mathrm{~m}^{2}$, and similar trends are also measured in hydrogen discharges in W7-X. The results obtained in this work cannot be explained by a drift-based or two-point model developed for the tokamak scrape-off layer, e.g., the dependence of the wetted area on $P_{S O L}$ is stronger. In case of the two-point model, when assuming constant transport coefficients $(\chi$, $\mathrm{D}, \kappa)$, the opposite relation is observed. The wetted area increases with the plasma temperature. Neither do we see a relation of $A_{w}$ to the plasma density, which was predicted by the two point model. This could imply that the thermal diffusivity is not constant and is increasing with the SOL power. But this needs further investigation.

The scaling of wetted areas for the 3D island structure in Wendelstein $7-\mathrm{X}$ is very beneficial for future fusion reactors, which will operate at much higher heating powers and SOL power but only slightly higher densities. Results in tokamaks ${ }^{3}$ show opposite to the results here a weak dependency on the separatrix power $\left(\mathrm{P}_{S O L}^{0.11 \pm 0.09}\right)$ and for some machines, a rather strong inverse scaling with the density.

Tokamaks operate with a poloidal divertor with a more simple geometry, which can be analyzed in one poloidal plane if no 3D perturbation is applied. Using formula 1 with $\lambda_{i n t}=\lambda_{q}+1.64 S^{12}$, where S stands for divertor broadening, the wetted area for other machines can be calculated. For a comparison, a medium-sized tokamak, ASDEX Upgrade (AUG), and a larger tokamak, Joint Europe Torus (JET), are used.

In AUG for L-mode discharges in Hydrogen, this leads to values of 0.27 to $0.62 \mathrm{~m}^{2}\left(\lambda_{q}=3.4-7.45 \mathrm{~mm}\right.$; $\mathrm{S}=0.4$ $1.16 \mathrm{~mm})$ for the outer target and 0.13 to $0.2 \mathrm{~m}^{2}\left(\lambda_{q}=\right.$ $\left.1.3-2.7 \mathrm{~mm} ; \mathrm{S}=0.5-0.8 \mathrm{~mm}, f_{\text {tor }}=0.8, f_{x}=8.2\right)$ for the inner target ${ }^{12}$, giving a total wetted area of 0.4 to $0.8 \mathrm{~m}^{2}$.

Values of the wetted area of up to $0.51 \mathrm{~m}^{2}\left(\lambda_{q}=3.1 \mathrm{~mm}\right.$; $\mathrm{S}=2 \mathrm{~mm} ; f_{x}=10^{3,16}$ ) are calculated for the outer target in deuterium H-mode discharges. Taking a similar ratio as given in the L-mode discharges, for outer and inner target result in a total wetted area in $\mathrm{H}$-mode in $\mathrm{AUG}$ of up to $0.70 \mathrm{~m}^{2}$.

For JET, the wetted area for the outer target is reported with values between 0.4 and $0.75 \mathrm{~m}^{2}$ in $\mathrm{H}$-mode for interELM programs ${ }^{11,17}$. For L-mode discharges in JET with the given data in ${ }^{18}\left(\lambda_{q}=3.5-6.5 \mathrm{~mm}, \mathrm{~S}=6.1 \mathrm{~mm}, f_{x}=5\right)$ lead to wetted area on the outer target of 0.91 to $1.12 \mathrm{~m}^{2}$. Assuming a similar ratio between the outer and inner target as in AUG results in a total wetted area in JET of 0.57 to $1.06 \mathrm{~m}^{2}$ for the inter-ELM regime in $\mathrm{H}$-mode and 1.3 to $1.59 \mathrm{~m}^{2}$ for L-mode. All this data is summarized in table I together with the calculated area of the last closed flux surface $A_{S O L}$ and the ratio of both areas.

The ratio of the areas is an indicator for the focussing of the available SOL area towards the used divertor area. Lower values are indicators for lower heat flux and better spreading of the energy in the SOL. W7-X operates with area ratios similar to AUG, but with total wetted areas in the range of JET L-mode. This difference is probably due to the 3D edge magnetic topology with its large island chains and connection lengths in the edge of the order of $100 \mathrm{~m}^{19}$. The heat flux can be spread out more by perpendicular transport processes, while the plasma particles flow along the long magnetic field lines. 


\begin{tabular}{|c|c|c|c|c|}
\hline Machine & Gas & $\begin{array}{c}A_{S O L} \\
{\left[\mathrm{~m}^{2}\right]}\end{array}$ & $\begin{array}{c}\text { wetted area } \\
\qquad\left[\mathrm{m}^{2}\right]\end{array}$ & $\frac{A_{S O L}}{A_{w}}$ \\
\hline AUG(L-Mode $)^{12}$ & $\mathrm{H}$ & 50 & 0.80 & 63 \\
\hline AUG $(\text { H-Mode })^{3,16}$ & $\mathrm{D}$ & 50 & 0.70 & 71 \\
\hline $\mathrm{W} 7-\mathrm{X}$ & $\mathrm{He}$ & 115 & 1.49 & 77 \\
\hline $\mathrm{W} 7-\mathrm{X}$ & $\mathrm{H}$ & 115 & 1.68 & 68 \\
\hline JET (L-mode) $)^{18}$ & $\mathrm{H}$ & 178 & 1.59 & 112 \\
\hline JET (H-mode $)^{3,11,17}$ & $\mathrm{H}$ & 178 & 1.06 & 167 \\
\hline
\end{tabular}

TABLE I. Maximum wetted area of different machines for the typical operation regime.

In contrast to that, adding 3D structures to the edge of Tokamaks does not seem to affect the averaged $\lambda_{q}$ in L-mode ${ }^{20}$. It has to be mentioned that for both types of machines, the divertor concepts can be further tuned to enlarge the wetted areas. For tokamaks, the incidence angle can be decreased by increasing $\mathrm{f}_{x}$, resulting in up to $20 \%$ higher wetted areas with a risk of leading edges. W7-X can also move the $\mathrm{x}$-point and has configurations with longer connection lengths ${ }^{21}$. In these, the perpendicular transport contributes even stronger, resulting in larger wetted areas. The change of the SOL width with different configurations is shown in $^{22}$. Here we compared regular used divertor configurations.

In this paper, a definition of the wetted area for the stellarator Wendelstein 7-X is presented. It is shown that the island divertor concept of W7-X allows large wetted areas of up to $1.5 \mathrm{~m}^{2}$ (in standard configuration). Furthermore, the wetted area is increasing with the power in the SOL, which is very beneficial for future reactors.

\section{ACKNOWLEDGMENTS}

This work has been carried out within the framework of the EUROfusion Consortium and has received funding from the Euratom research and training program 20142018 and 2019-2020 under grant agreement No 633053. The views and opinions expressed herein do not necessarily reflect those of the European Commission.

\footnotetext{
${ }^{1}$ T. Klinger, T. Andreeva, S. Bozhenkov, C. Brandt, R. Burhenn, B. Buttenschön, G. Fuchert, B. Geiger, O. Grulke, H. Laqua, et al., Nuclear Fusion 59, 112004 (2019), URL https://doi. org $/ 10.1088 \% 2 \mathrm{~F} 1741-4326 \% 2 \mathrm{Fab} 03 \mathrm{a} 7$.

${ }^{2}$ A. Loarte, B. Lipschultz, A. Kukushkin, G. Matthews, P. Stangeby, N. Asakura, G. Counsell, G. Federici, A. Kallenbach, K. Krieger, et al., Nuclear Fusion 47, S203 (2007), URL https://doi.org/10.1088\%2F0029-5515\%2F47\%2F6\%2Fs04.

${ }^{3}$ T. Eich, A. Leonard, R. Pitts, W. Fundamenski, R. Goldston, T. Gray, A. Herrmann, A. Kirk, A. Kallenbach, O. Kardaun, et al., Nuclear Fusion 53, 093031 (2013), URL https://doi. org/10.1088\%2F0029-5515\%2F53\%2F9\%2F093031.

${ }^{4}$ R. König, P. Grigull, K. McCormick, Y. Feng, J. Kisslinger, A. Komori, S. Masuzaki, K. Matsuoka, T. Obiki, N. Ohyabu, et al., Plasma Phys. Control. Fusion 44, 2365 (2002).
}

${ }^{5}$ Y. Feng, M. Kobayashi, F. Sardei, S. Masuzaki, J. Kisslinger, T. Morisaki, P. Grigull, H. Yamada, K. McCormick, N. Ohyabu, et al., Nuclear Fusion 49 (2009), URL https://doi.org/10. 1088/0029-5515/49/9/095002.

${ }^{6}$ H.-S. Bosch, R. Wolf, T. Andreeva, J. Baldzuhn, D. Birus, T. Bluhm, T. Bräuer, H. Braune, V. Bykov, A. Cardella, et al., Nuclear Fusion 53, 126001 (2013), URL https://doi.org/10. 1088\%2F0029-5515\%2F53\%2F $12 \% 2$ F 126001 .

${ }^{7}$ M. Jakubowski, P. Drewelow, J. Fellinger, A. Puig Sitjes, G. Wurden, A. Ali, C. Biedermann, B. Cannas, D. Chauvin, M. Gamradt, et al., Review of Scientific Instruments 89, $10 \mathrm{E} 116$ (2018), URL https://aip.scitation.org/doi/10.1063/1.5038634.

${ }^{8}$ A. Herrmann, 28th EPS conference on Controlled Fusion and Plasma Physics 25A, 2109 (2001), URL http://hdl.handle. net/11858/00-001M-0000-0027-49DD-7.

${ }^{9}$ B. Sieglin, M. Faitsch, A. Herrmann, B. Brucker, T. Eich, L. Kammerloher, and S. Martinov, REVIEW OF SCIENTIFIC INSTRUMENTS 86, 113502 (2015), URL https://doi.org/10. 1063/1.4935580

${ }^{10}$ Y. Gao, M. W. Jakubowski, P. Drewelow, F. Pisano, A. P. Sitjes, H. Niemann, A. Ali, B. Cannas, and W.-X. Team, Nuclear Fusion 59, 066007 (2019), URL https ://doi.org/10.1088\% $2 \mathrm{~F} 1741-4326 \% 2 \mathrm{Fab} 0 \mathrm{f} 49$.

${ }^{11}$ T. Eich, H. Thomsen, W. Fundamenski, G. Arnoux, S. Brezinsek, S. Devaux, A. Herrmann, S. Jachmich, J. Rapp, and JET-EFDA contributors, Journal of Nuclear Materials 415, S856 (2011), URL https://doi.org/10.1016/j.jnucmat.2010.11.079.

${ }^{12}$ B. Sieglin, T. Eich, M. Faitsch, A. Hermann, A. Scarabosio, and the ASDEX Upgrade Team, Plasma Physics and Controlled Fusion 58, 055015 (2016), URL https://doi.org/10. 1088/0741-3335/58/5/055015.

${ }^{13}$ K. C. Hammond, Y. Gao, M. Jakubowski, C. Killer, H. Niemann, L. Rudischhauser, A. Ali, T. Andreeva, B. D. Blackwell, K. J. Brunner, et al., Plasma Physics and Controlled Fusion 61, 125001 (2019), URL https://doi.org/10.1088\%2F1361-6587\% $2 \mathrm{Fab} 4825$

${ }^{14}$ Y. Feng, M. Kobayashi, T. Lunt, and D. Reiter, Plasma Physics and Controlled Fusion 53, 024009 (2011), URL https://doi. org $/ 10.1088 \% 2 \mathrm{~F} 0741-3335 \% 2 \mathrm{~F} 53 \% 2 \mathrm{~F} 2 \% 2 \mathrm{~F} 024009$.

${ }^{15}$ R. Goldston, Nuclear Fusion 52, 013009 (2011), URL https: //doi.org/10.1088/0029-5515/52/1/013009.

${ }^{16}$ T. Eich, B. Sieglin, A. Scarabosio, A. Herrmann, A. Kallenbach, G. Matthews, S. Jachmich, S. Brezinsek, M. Rack, and R. Goldston, Journal of Nuclear Materials 438, S72 (2013), ISSN 0022-3115, proceedings of the 20th International Conference on Plasma-Surface Interactions in Controlled Fusion Devices, URL http://www.sciencedirect.com/science/article/ pii/S0022311513000196.

${ }^{17}$ S. Jachmich, G. Arnoux, S. Brezinsek, S. Devaux, T. Eich, W. Fundamenski, C. Giroud, H. Koslowski, Y. Liang, E. de la Luna, et al., Journal of Nuclear Materials 415, S894 (2011), URL https://doi.org/10.1016/j.jnucmat.2010.12.015.

${ }^{18}$ A. Scarabosio, T. Eich, A. Herrmann, and B. Sieglin, Journal of Nuclear Materials 438, S426 (2013), ISSN 0022-3115, proceedings of the 20th International Conference on Plasma-Surface Interactions in Controlled Fusion Devices, URL http://www. sciencedirect.com/science/article/pii/S0022311513000949.

${ }^{19}$ Y. Feng, F. Sardei, P. Grigull, K. McCormick, J. Kisslinger, D. Reiter, and Y. Igitkhanov, Plasma Physics and Controlled Fusion 44, 611 (2002), URL https://doi.org/10.1088\% $2 \mathrm{~F} 0741-3335 \% 2 \mathrm{~F} 44 \% 2 \mathrm{~F} 5 \% 2 \mathrm{~F} 308$.

${ }^{20}$ M. Faitsch, B. Sieglin, T. Eich, A. Herrmann, and W. S. and, Plasma Physics and Controlled Fusion 59, 095006 (2017), URL https://doi .org/10.1088\%2F1361-6587\%2Faa75e7.

${ }^{21} \mathrm{P}$. Sinha, H. Hölbe, T. S. Pedersen, S. Bozhenkov, and W7-X Team, Nuclear Fusion 58, 016027 (2017), URL https://doi. org/10.1088/1741-4326/aa9496.

${ }^{22}$ C. Killer, O. Grulke, P. Drews, Y. Gao, M. Jakubowski, A. Knieps, D. Nicolai, H. Niemann, A. P. Sitjes, and G. S. and, Nuclear Fusion 59, 086013 (2019), URL https://doi.org/10. 
1088\%2F $1741-4326 \% 2 F a b 2272$ 